\title{
An Enhanced Approach for Low Bit Rate Image Compression Using Autoregressive Modeling
}

\author{
H.Shalini ${ }^{1}$, A.Vamsidhar ${ }^{2}$, K.S.N.V.Someswara Rao ${ }^{3}$, G.Santhi Kumari ${ }^{4}$ \\ ${ }^{I}$ (Department of E.C.E, M.tech-SSP,DIET College of Engineering, Visakhapatnam,India) \\ ${ }^{2}$ (Department of E.C.E, Assoc.Professor, DIET College, Visakhapatnam,India) \\ ${ }^{3}$ (Department of E.C.E, Asst.Professor, DIET College, Visakhapatnam,India) \\ ${ }^{4}$ (Department of E.C.E, Asst.Professor, LIET College, Vizianagaram,India)
}

\begin{abstract}
In this paper, we are going to use a practical approach of uniform down sampling in image space and yet making the sampling adaptive by spatially varying, directional low-pass pre-filtering. The resulting down-sampled pre-filtered image remains a conventional square sample grid, and, thus, it can be compressed, transmitted without any change to current image coding standards and systems. The decoder first decompresses the low-resolution image and then up-converts it to the original resolution in a constrained least squares restoration process, using a 2-D piecewise autoregressive model and the knowledge of directional low-pass prefiltering. The proposed compression approach of collaborative outperforms JPEG 2000 in PSNR measure at low to medium bit rates and achieves superior visual quality, as well. The superior low bit-rate performance of the Collaborative Adaptive Down-sampling and Upconversion (CADU) approach seems to suggest that oversampling not only wastes hardware resources and energy, and it could be counterproductive to image quality given a tight bit budget.
\end{abstract}

Keywords: Autoregressive modeling, compression standards, image restoration, image up conversion, low bit-rate image compression.

\section{Introduction}

The term digital image refers to processing of a two dimensional picture by a digital computer. In a broader context, it implies digital processing of any two dimensional data. A digital image is an array of real or complex numbers represented by a finite number of bits. An image given in the form of a transparency, slide, photograph or an X-ray is first digitized and stored as a matrix of binary digits in computer memory. This digitized image can then be processed and/or displayed on a high-resolution television monitor. For display, the image is stored in a rapid-access buffer memory, which refreshes the monitor at a rate of 25 frames per second to produce a visually continuous display.

Image enhancement techniques were studied the proper enhancement techniques for the specific application was found out. Various enhancement methods were implemented. The frames captured were enhanced using these methods and a later this was done in real time. It was found that for acquiring large number of frames at a faster rate Matlab to $\mathrm{C}$ interfacing was required. An interface was created and Matlab functions were called from $\mathrm{C}$ environment. This inturn was used to acquire real time images. The basic principles involved in image storage, techniques involved in image compression were studied. The image compression algorithms namely JPEG, JPEG2000 and MPEG-4 were studied in detail. JPEG algorithm was understood and implemented[1] on image sub blocks and on the entire image. Various aspects of the algorithm such as effect of DC coefficient, blocking artifacts etc was studied and implemented in real time. The algorithm was implemented in real time in Matlab-7 and the results analyzed. The advantages and short comings of this algorithm were studied.The complete algorithm of JPEG2000 was studied. The short comings of JPEG were eliminated using JPEG2000. The algorithm was implemented in real time in Martlab-7.The advantages and key features of this algorithm were studied and implemented. The tradeoffs in both JPEG and JPEG2000 were also studied[4,10]. An equivalent $C$ code for the JPEG algorithm was developed and it was successfully compiled and executed. This was dumped on a Blackfinn[3] DSP processor and a hardware model for a real time image acquisition and compression was set up. This was done by interfacing video to the Blackfin processor and also to the PC.Thus a complete system (A hardware model) for a real time image acquisition and compression was set up. The modifications if any can be simulated in Matlab-7 and if the results are improved can be incorporated on the hardware model by making equivalent changes in the $\mathrm{C}$ code. This system (algorithm) has important application in the modern world such as Telemedicine and other communication applications 


\subsection{The Image Processing System}

A typical digital image processing system is given in figure1.

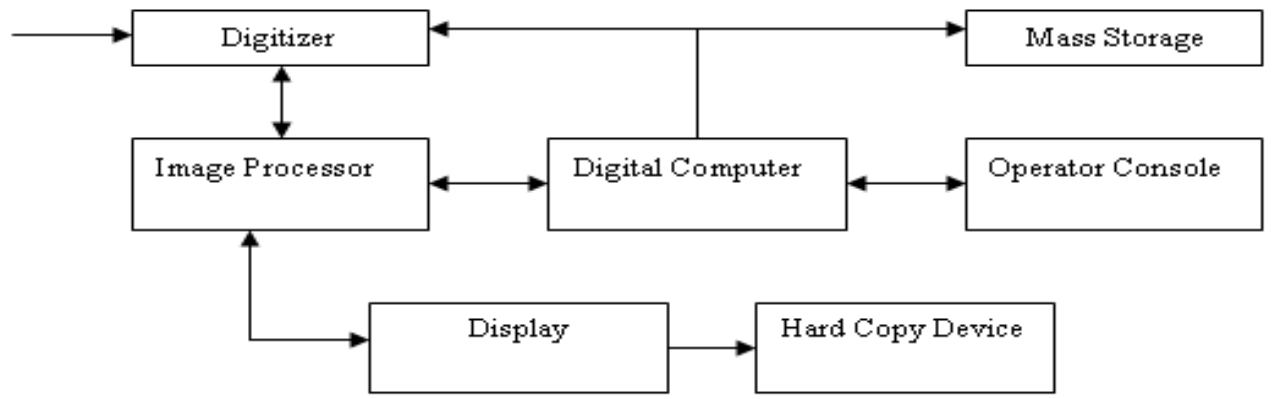

Fig 1. Block Diagram of a Typical Image Processing System

\subsubsection{Digitizer}

A digitizer converts an image into a numerical representation suitable for input into a digital computer. Some common digitizers are

- Microdensitometer

- Flying spot scanner

- Image dissector

- Videocon camera

- Photosensitive solid- state arrays.

\subsubsection{Image Processor}

An image processor does the functions of image acquisition, storage, preprocessing, segmentation, representation, recognition and interpretation and finally displays or records the resulting image. The following block diagram gives the fundamental sequence involved in an image processing system as shown in figure 2.

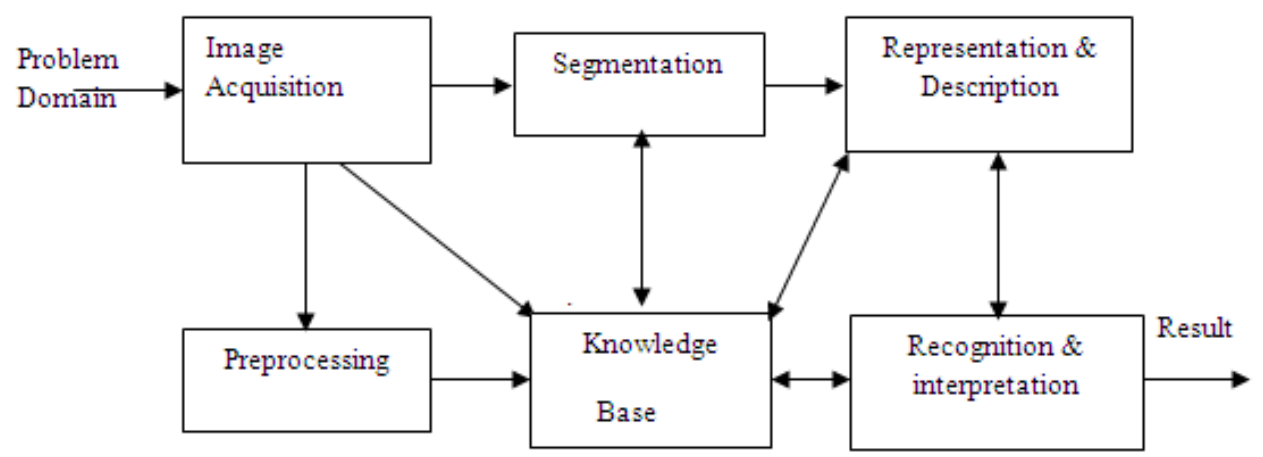

Fig 2. Block Diagram of Fundamental Sequence involved in an image Processing system

As detailed in the diagram, the first step in the process is image acquisition by an imaging sensor in conjunction with a digitizer to digitize the image. The next step is the preprocessing step where the image is improved being fed as an input to the other processes. Preprocessing typically deals with enhancing, removing noise, isolating regions, etc. Segmentation partitions an image into its constituent parts or objects. The output of segmentation is usually raw pixel data, which consists of either the boundary of the region or the pixels in the region themselves. Representation is the process of transforming the raw pixel data into a form useful for subsequent processing by the computer. Description deals with extracting features that are basic in differentiating one class of objects from another. Recognition assigns a label to an object based on the information provided by its descriptors. Interpretation involves assigning meaning to an ensemble of recognized objects. The knowledge about a problem domain is incorporated into the knowledge base. The knowledge base guides the operation of each processing module and also controls the interaction between the modules. Not all modules need be necessarily present for a specific function. The composition of the image processing system depends on its application. The frame rate of the image processor is normally around 25 frames per second. 


\subsubsection{Digital Computer}

Mathematical processing of the digitized image such as convolution, averaging, addition, subtraction, etc. are done by the computer.

\subsubsection{Mass Storage}

The secondary storage devices normally used are floppy disks, CD ROMs etc.

\subsubsection{Hard Copy Device}

The hard copy device is used to produce a permanent copy of the image and for the storage of the software involved.

\subsubsection{Operator Console}

The operator console consists of equipment and arrangements for verification of intermediate results and for alterations in the software as and when require. The operator is also capable of checking for any resulting errors and for the entry of requisite data.

\section{Uniform Down-Sampling With Adaptive Directional Prefiltering}

Out of practical considerations, we make a more compact representation of an image by decimating every other row and every other column of the image. This simple approach has an operational advantage that the down-sampled image remains a uniform rectilinear grid of pixels and can readily be compressed by any of existing international image coding standards. To prevent the down-sampling process from causing aliasing artifacts, it seems necessary to low-pass pre filter an input image to half of its maximum frequency $f_{\max }$ However, on a second reflection, one can do somewhat better. In areas of edges, the 2-d spectrum of the local image signal is not isotropic[2,4]. Thus, we seek to perform adaptive sampling, within the uniform downsampling framework, by judiciously smoothing the image with directional low-pass pre filtering prior to down sampling. The proposed CADU compression system as shown in figure 3.

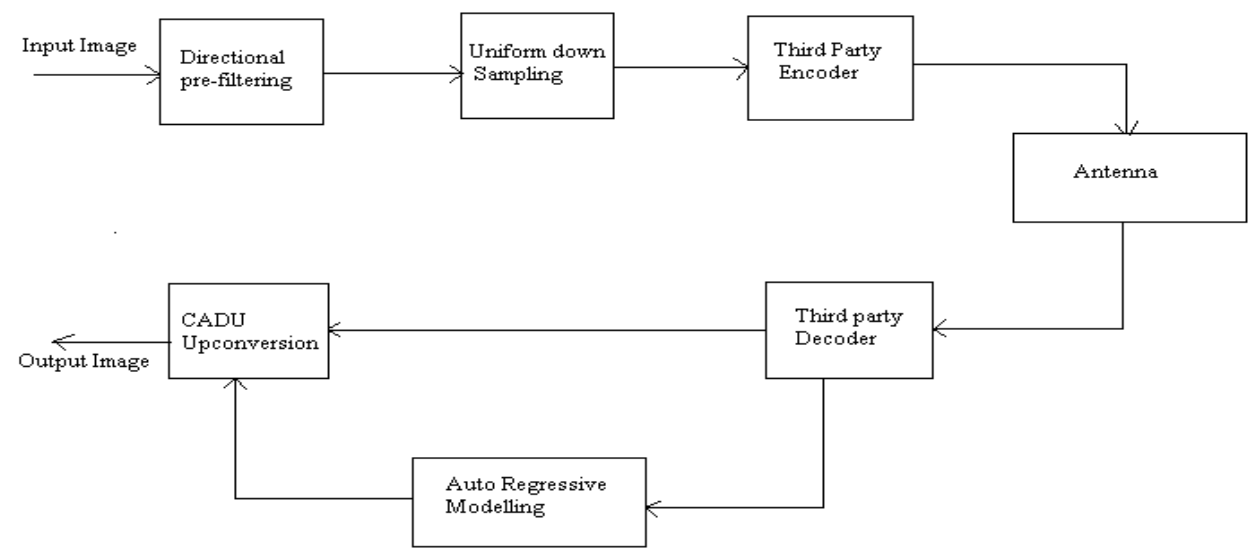

Fig 3.Block Diagram of The Proposed CADU Image Compression System

In the directional pre filtering step, the CADU encoder first computes the gradient at the sampled position. Despite its simplicity, the CADU compression approach via uniform down-sampling[7] is not inherently inferior to other image compression techniques in rate-distortion performance, as long as the target bit rate is below a threshold. The argument is based on the classical water-filling principle in rate-distortion theory. To encode a set of $\mathrm{K}$ Independent Gaussian random variables $\left\{\mathrm{X} 1, \mathrm{X} 2, \ldots, \mathrm{X}_{\mathrm{k}}\right\}, \mathrm{Xk} \mathrm{N}(0, \sigma \mathrm{k})$ the rate distortion bounds, when the total bit rate being $R=\sum_{K-1}^{K} R_{K}$ and the total mean-squares distortion being $\mathrm{D}=\sum_{K-1}^{K} D_{K}$, are given by

$$
\begin{aligned}
& R(D)=\sum_{K=1}^{K} \max \left\{\left[0, \frac{1}{2} \log _{2} \frac{\sigma^{2} k}{\tau}\right\}\right. \\
& D(K)=\sum_{K=1}^{K} \min \left\{\tau, \sigma_{k}^{2}\right\}
\end{aligned}
$$




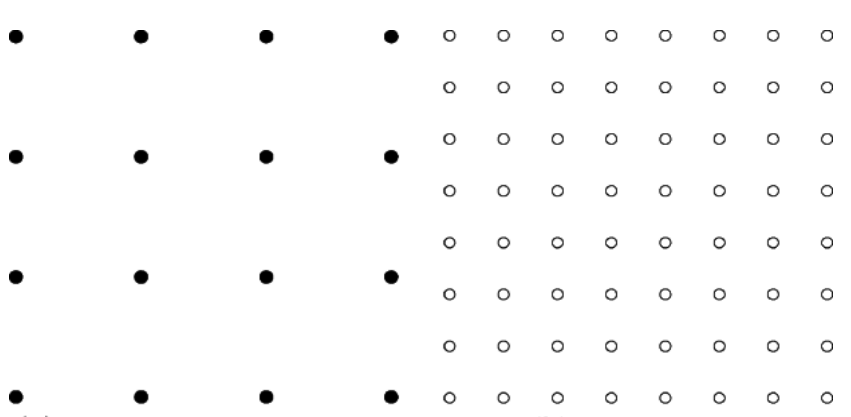

(a) Downsampled prefiltered image

(b) Original image

Fig 4. Relationship between the down-sampled pre filtered image and the original image.

The illustrated kernel size of the filter is 3. Low-resolution pixel [black dots in (a)] is the filtered value of the corresponding nine original pixels [white dots in (b)]. (a) Down sampled pre filtered image; (b) original image.

\subsection{Module:}

Module 1: Decompression of low -resolution image.

Module 2: Up conversion of the image to its resolution by PAR.

Module 3: Reverse the directional low-pass profiteering operation of the encoder.

\subsection{1 .Module Description}

The CADU decoder first decompresses the low resolution image and then upconverts it to the original resolution in constrained least squares restoration process, using a 2-D piecewise autoregressive model (PAR) [2]and by reversing the directional low-pass pre filtering operation of the encoder. Two-dimensional autoregressive modeling was a known effective technique of predictive image coding For the CADU decoder[1]; the PAR model plays a role of adaptive non causal predictor. The CADU approach is very novel and unique that the predictor is only used at the decoder side, and the non causal predictive decoding is performed in collaboration with the pre filtering of the encoder.

\subsubsection{Module 1:}

The CADU image compression technique, although operating on down-sampled images, obtains some of the best PSNR results and visual quality at low to medium bit rates. CADU outperforms the JPEG 2000 standard, even though the latter is fed images of

Higher resolution and is widely regarded as an excellent low bit-rate image codec. Since the down sampled image has the conventional form of square pixel grid and can be fed directly to any existing image codec, standard or proprietary, the CADU upconversion process[6] is entirely up to the decoder the proposed CADU image coding approach can work in tandem with any third party image/video compression techniques. This flexibility makes standard compliance a non issue for the new CADU method. We envision that CADU becomes a useful enhancer of any existing image compression standard for improved low bit-rate performance.

\subsubsection{Module 2:}

Out of practical considerations, we make a more compact representation of an image by decimating every other row and every other column of the image. This simple approach has an operational advantage that the down-sampled image remains a uniform

rectilinear grid of pixels and can readily be compressed by any of existing international image coding standards. To prevent the down-sampling process from causing aliasing artifacts, it seems necessary to low-pass prefilter [8] an input image to half of its maximum frequency fmax. In areas of edges, the 2-D spectrum of the local image signal is not isotropic. Thus, we seek to perform adaptive sampling, within the uniform downsampling framework, by smoothing the image with directional low-pass prefiltering prior to down-sampling.

\subsubsection{Module 3:}

Because the down-sampled image is only a small fraction of the original size, CADU greatly reduces the encoder complexity, regardless what third-party codec is used in conjunction. This property allows the system to shift the computation burden from the encoder to decoder, making CADU a viable asymmetric compression solution when the encoder is resource deprived. Furthermore, the superior low bit-rate performance of the CADU approach seems to suggest that a camera of unnecessarily high resolution can ironically produce inferior images than a lower resolution camera, if given a tight bit budget. This rather counter-intuitive 
observation should be heeded when one designs visual communication devices/systems with severe constraints of energy and bandwidth.

\section{Constrained Least Squares Conversion Withautoregressive Modeling}

In this section, we develop the decoder of the CADU image compression system. We formulated the constrained least square problem using two PAR models of order 4 each: the model of parameters a and the model of parameters . The two PAR models characterize the axial and diagonal correlations, respectively, as depicted in Fig.4. These two models act, in a predictive coding perspective, as non causal adaptive predictors. This gives rise to an interesting interpretation of the CADU decoder: adaptive non causal predictive decoding constrained by the pre filtering operation of the encoder. Therefore the par model parameters $\mathbf{a}$ and $\mathbf{b}$ can be estimated from the decoded image by solving the following least square estimation

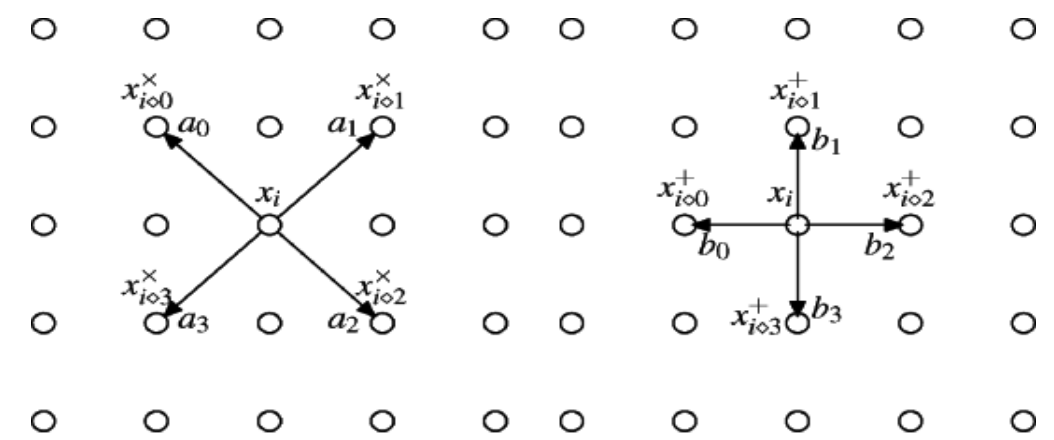

(a)

(b)

Fig 5. Sample relationships with PAR model parameters (a) $\mathbf{a}=(\mathrm{a} 0, \mathrm{a} 1, \mathrm{a} 2, \mathrm{a} 3),(\mathrm{b}) \mathbf{b}=(\mathrm{b} 0, \mathrm{~b} 1, \mathrm{~b} 2, \mathrm{~b} 3)$

$$
\begin{aligned}
& \hat{\boldsymbol{a}}=\arg \min _{\boldsymbol{a}}\left\{\sum_{i \in W}\left(y_{i}-\sum_{0 \leq t \leq 3} a_{t} y_{i \circ t}^{\times}\right)^{2}\right\} \\
& \hat{\boldsymbol{b}}=\arg \min _{\boldsymbol{b}}\left\{\sum_{i \in W}\left(y_{i}-\sum_{0 \leq t \leq 3} b_{t} y_{i \circ t}^{+}\right)^{2}\right\}
\end{aligned}
$$

The closed form solution for the above equations is:

$$
\begin{aligned}
& \hat{\boldsymbol{a}}=\left(\mathbf{A}^{T} \mathbf{A}\right)^{-1} \mathbf{A}^{T} \mathbf{v} \\
& \hat{\boldsymbol{b}}=\left(\mathbf{B}^{T} \mathbf{B}\right)^{-1} \mathbf{B}^{T} \mathbf{v}
\end{aligned}
$$

The constrained least square problem can be converted to the following unconstrained least square problem:

$$
\begin{aligned}
& \min _{\boldsymbol{x}}\left\{\xi^{\times}\right. \sum_{i \in W}\left(x_{i}-\sum_{t=0}^{3} a_{t} x_{i \diamond t}^{\times}\right)^{2}+ \\
&\left.\xi^{+} \sum_{i \in W}\left(x_{i}-\sum_{t=0}^{3} b_{t} x_{i \diamond t}^{+}\right)^{2}+\lambda(\boldsymbol{x} * \boldsymbol{h}-\boldsymbol{y})^{2}\right\}
\end{aligned}
$$

To solve the above equation we rewrite equation in matrix form:

$$
\min _{\boldsymbol{x}}\left\{(\mathbf{C} \boldsymbol{x}-\mathbf{d})^{2}\right\}
$$

Where $\mathbf{C}$ and $\mathbf{d}$ are composed of $\mathbf{a}, \mathbf{b}, \boldsymbol{\lambda}, \mathbf{h}$, and the decoded pixels $\mathbf{y}$.The CADU system design is asymmetric: the encoder is a simple and inexpensive process, while the decoder involves solving a rather largescale optimization problem described. The computation bottleneck is in inverting an $\mathbf{n} \times \mathbf{n}$ matrix, where $\mathbf{n}$ is the number of pixels to be jointly recovered. Instead of inverting the matrix CTC directly, we solve numerically via 
differentiation using the conjugate gradient method. The solution is guaranteed to be globally optimal for the objective function is convex.

\section{Experimental Results}

Extensive experiments were carried out to evaluate the proposed image coding method, in both PSNR and subjective quality. We compared the CADU method with the adaptive downsampling-based image codec proposed by Lin and Dong. The latter was reportedly the best among all previously published downsamplinginterpolation image codecs[7], in both objective and subjective quality. Note that all existing image codecs of this type were developed for DCT-based image compression, whereas the CADU method is applicable to wavelet-based codecs as well. Therefore, we also include in our comparative study JPEG 2000, the quincunx coding method [9], and the method of uniform down-sampling at the encoder and bicubic interpolation at the decoder. The bicubic method in the comparison group and the CADU method used the same simple encoder: JPEG 2000 coding of uniformly down-sampled prefiltered image[8]. The difference is in the upconversion process: the former method performed bicubic image interpolation followed by a deconvolution step using Weiner filter to reverse the prefiltering, instead of solving a constrained least squares image restoration problem driven by autoregressive models as described in the proceeding section

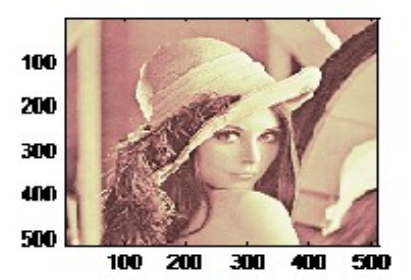

(a)

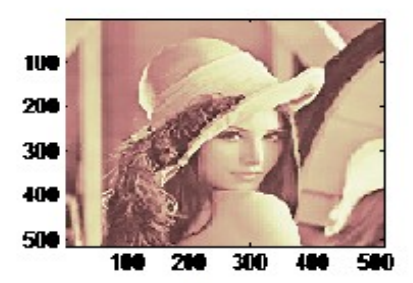

(d)

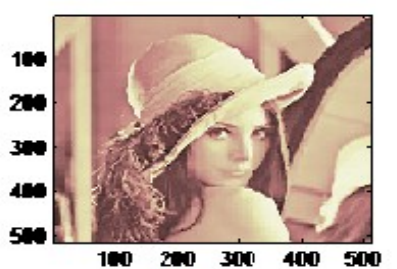

(b)

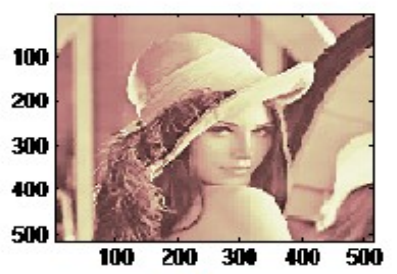

(e)

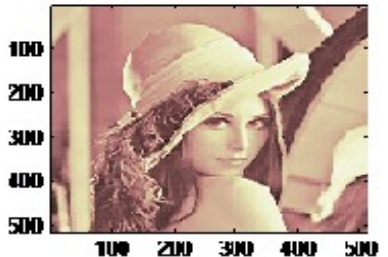

(c)

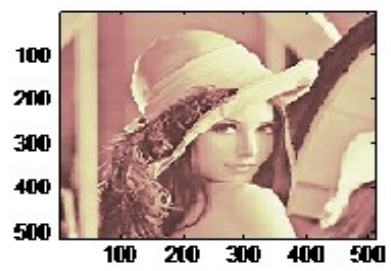

(f)

Fig 6.Comparison of different methods at 0.2 bpp. (a) JPEG; (b) Method ; (c) J2K; (d) CADU-JPG; (e) BicubicJ2K; (f) CADU-J2K;

The superior visual quality of the CADU-J2K method is due to the good fit of the piecewise autoregressive model to edge structures and the fact that human visual system is highly sensitive to phase errors in reconstructed edges We believe that the CADU-J2K image coding approach of down-sampling with directional pre- filtering at the encoder and edge-preserving up conversion at the decoder offers an effective and practical solution for subjective image coding. Some viewers may find that JPEG 2000 produces somewhat sharper edges compared with CADU-J2K, although at the expense of introducing more and worse artifacts. However, one can easily tip the quality balance in visual characteristics to favor CADU-J2K by performing an edge enhancement of the results of CADU-J2K. Some sample results of JPEG 2000 and CADU-J2K at the bit rate of 0.2 bpp after edge enhancement. For better judgment these images should be compared with their counterparts. As expected, the high-pass operation of edge enhancement magnifies the structured noises accompanying edges in images of JPEG2000. In contrast, edge enhancement sharpens the images of CADU$\mathrm{J} 2 \mathrm{~K}$ without introducing objectionable artifacts, which further improves the visual quality.

The CADU-J2K decoder has much higher complexity than the decoder based on bicubic interpolation. A close inspection of the reconstructed images by the CADU-J2K decoder and the bicubic method reveals that the two methods visually differ only in areas of edges. Therefore, an effective way of expediting the CADU-J2K decoder is to invoke least squares noncausal predic- tive decoding, which is the computation bottleneck of CADU, only in regions ofhigh activity, and resort to fast bicubic inter- polation in smooth regions[9]. If a decoder is severely constrained by computation resources, it can perform bicubic interpolation everywhere in lieu of the CADU restoration process. Such a re- source scalability of the decoder is desired in application scenarios when decoders of diverse capabilities are to work with the same code stream. 


\section{Conclusions}

This paper deals with new, standard-compliant approach of coding uniformly down-sampled images, which outperforms JPEG 2000 in both PSNR and visual quality at low to modest bit. Hence the proposed method is not only a simple, practical algorithm, but also an effective algorithm. When compared with the previous results, with this algorithm better results were obtained. The proposed approach says that a lower sampling rate can actually produce higher quality images at certain bit rates. By feeding the standard methods down sampled images, the new approach reduces the workload and energy consumption of the encoders, which is important for wireless visual communication.

VI.FUTURE SCOPE This system(algorithm) has important application in the modern world such as Telemedicine and other communication applications.

\section{VI.FUTURE SCOPE}

- This system(algorithm) has important application in the modern world such as Telemedicine and other communication applications.

- CADU restoration process can be used to perform Bicubic interpolation

- Decoders of diverse capabilities can be made to work with the same code stream.

\section{REFERENCES}

[1]. E. Cands, “Compressive sampling,” in Proc. Int. Congr. Mathematics, Madrid, Spain, 2006, pp. 1433-1452. WU et al.: LOW BITRATE IMAGE COMPRESSION VIA ADAPTIVE DOWN-SAMPLING 561

[2]. X.Wu,K.U. Barthel, and W. Zhang, "Piecewise 2-D autoregression for predictive image coding," in Proc. IEEE Int. Conf. Image Processing, Chicago, IL, Oct. 1998, vol. 3, pp. 901-904.

[3]. X. Li and M. T. Orchard, “Edge-direted prediction for lossless compression of natural images,” IEEE Trans. Image Process., vol. 10, no. 6, pp. 813-817, Jun. 2001.

[4]. D. Santa-Cruz, R. Grosbois, and T. Ebrahimi, "Jpeg 2000 performance evaluation and assessment," Signal Process.: Image Commun., vol. 1, no. 17, pp. 113-130, 2002.

[5]. A. M. Bruckstein, M. Elad, and R. Kimmel, “Down-scaling for better transform compression," IEEE Trans. Image Process., vol. 12, no. 9, pp. 1132-1144, Sep. 2003.

[6]. Y. Tsaig, M. Elad, and P. Milanfar, "Variable projection for near-optimal filtering in low bit-rate block coders," IEEE Trans. Circuits Syst. Video Technol., vol. 15, no. 1, pp. 154-160, Jan. 2005.

[7]. W. Lin and D. Li, “Adaptive downsampling to improve image compression at low bit rates,” IEEE Trans. Image Process., vol. 15, no. 9 , pp. 2513-2521, Sep. 2006.

[8]. L. Gan, C. Tu, J. Liang, T. D. Tran, and K.-K. Ma, "Undersampled boundary pre-/post-filters for low bit-rate dct-based coders," IEEE Trans. Image Process., vol. 16, no. 2, pp. 428-441, Feb. 2007.

[9]. X. Zhang, X. Wu, and F. Wu, "Image coding on quincunx lattice with adaptive lifting and interpolation," in Proc. IEEE Data Compression Conf., Mar. 2007, pp. 193-202.

[10]. B. Zeng and A. N.Venetsanopoulos, “A jpeg-based interpolative image coding scheme,” in Proc. IEEE ICASSP, 1993, vol. 5, pp. 393-396.

[11]. D. Tabuman and M. Marcellin, JPEG2000: Image Compression Fundamentals, Standards and Parctice. Norwell, MA: Kluwer, 2002. 